\title{
Rate of metastability for Bruck's iteration of pseudocontractive mappings in Hilbert space
}

\author{
Daniel Körnlein and Ulrich Kohlenbach* \\ Department of Mathematics \\ Technische Universität Darmstadt \\ Schlossgartenstraße 7, 64289 Darmstadt, Germany
}

May 24, 2013

\begin{abstract}
We give a quantitative version of the strong convergence of Bruck's iteration schema for Lipschitzian pseudocontractions in Hilbert space.
\end{abstract}

Keywords: Accretive mappings, pseudocontractive mappings, fixed points, asymptotic regularity, metastability, proof mining.

Mathematics Subject Classification (2010): 47H06; 47H05; 47H10; 03F10

\section{Introduction}

Let $X$ be a normed linear space and $S \subseteq X$ be a subset of $X$. In 1967, Browder introduced an important generalization of the class of nonexpansive mappings, namely the pseudocontractive mappings $T: S \rightarrow S$ defined by

$$
\forall u, v \in S \forall \lambda>1((\lambda-1)\|u-v\| \leq\|(\lambda I-T)(u)-(\lambda I-T)(v)\|),
$$

where $I$ denotes the identity mapping.

Apart from being a generalization of nonexpansive mappings, the pseudocontractive mappings are also closely related to accretive operators, where an operator $A$ is called accretive if for every $u, v \in S$ and for all $s>0$,

$$
\|u-v\| \leq\|u-v+s(A u-A v)\| .
$$

Observe that $T$ is pseudocontractive if and only if $I-T$ is accretive. Therefore, any fixed point of $T$ is a root of the accretive operator $I-T$.

In a Hilbert space, $T$ is pseudocontractive iff

$$
\forall u, v \in S\left(\langle T u-T v, u-v\rangle \leq\|u-v\|^{2}\right)
$$

(see e.g. [5]).

In [4], Bruck introduced the following iteration schema for pseudocontractive mappings:

Definition 1.1 ( [4]). Let $C$ be a nonempty convex subset of a real normed space and let $T$ : $C \rightarrow C$ be a pseudocontraction. Let $\left(\lambda_{n}\right),\left(\theta_{n}\right)$ be sequences in $[0,1]$ with $\lambda_{n}\left(1+\theta_{n}\right) \leq 1$ for all $n \in \mathbb{N}$. The Bruck iteration scheme with starting point $x_{1} \in C$ is defined as

$$
x_{n+1}=\left(1-\lambda_{n}\right) x_{n}+\lambda_{n} T x_{n}-\lambda_{n} \theta_{n}\left(x_{n}-x_{1}\right) .
$$

\footnotetext{
${ }^{*}$ The authors have been supported by the German Science Foundation (DFG Project KO 1737/5-1, 1737/5-2).
} 
Among many other things, Bruck showed that in Hilbert spaces and for bounded closed and convex subsets $C$ this iteration strongly converges for so-called acceptably paired sequences $\left(\lambda_{n}\right),\left(\theta_{n}\right)$. Moreover the limit is a fixed point of $T$ provided that $T$ is demicontinuous (in addition to being pseudocontractive).

In [6], it is shown that Bruck's iteration (with more natural conditions on $\left(\lambda_{n}\right),\left(\theta_{n}\right)$ ) is asymptotically regular, i.e.

$$
\left\|x_{n}-T\left(x_{n}\right)\right\| \stackrel{n \rightarrow \infty}{\rightarrow} 0
$$

in arbitrary Banach spaces provided that $T$ is a Lipschitzian pseudocontractive mapping which still includes the important class of strictly pseudocontrative mappings in the sense of Browder and Petryshyn [3] (see [5]).

Definition 1.2 ( $[6])$. The real sequences $\left(\lambda_{n}\right)$ and $\left(\theta_{n}\right)$ in $(0,1]$ are said to satisfy the ChidumeZegeye conditions if

1. $\lim _{n \rightarrow \infty} \theta_{n}=0$;

2. $\sum_{n=1}^{\infty} \lambda_{n} \theta_{n}=\infty$;

3. $\forall \varepsilon>0 \exists m \in \mathbb{N} \forall n \geq m\left(\lambda_{n} \leq \theta_{n} \varepsilon\right)$;

4. $\forall \varepsilon>0 \exists m \in \mathbb{N} \forall n \geq m\left(\frac{\left|\frac{\theta_{n-1}}{\theta_{n}}-1\right|}{\lambda_{n} \theta_{n}} \leq \varepsilon\right)$;

5. $\lambda_{n}\left(1+\theta_{n}\right) \leq 1$ for all $n \in \mathbb{N}$.

Notation: For $T: C \rightarrow C$ let $F(T)$ be the set of fixed points of $T$.

Theorem 1.3 ( [6]). Let $C$ be a nonempty closed convex subset of a real Banach space $X$. Let $T: C \rightarrow C$ be a Lipschitz pseudocontractive map with Lipschitz constant $L$ and $F(T) \neq \emptyset$. Let $\left(x_{n}\right)$ be the Bruck iteration with starting point $x_{1} \in C$, where the parameters $\left(\lambda_{n}\right)$ and $\left(\theta_{n}\right)$ satisfy the Chidume-Zegeye conditions. Then $\left\|x_{n}-T x_{n}\right\| \rightarrow 0$ as $n \rightarrow \infty$.

Remark 1.4. Instead of $F(T) \neq \emptyset$ one can also assume that $C$ is bounded.

In fact, Theorem 1.3 is shown as a consequence of the fact that $\left\|x_{n}-z_{n-1}\right\| \rightarrow 0$, where $z_{n}$ is the unique point (whose existence is guaranteed by [12]) satisfying

$$
z_{n}=t_{n} T\left(z_{n}\right)+\left(1-t_{n}\right) x_{1}, \text { where } t_{n}:=\frac{1}{1+\theta_{n}} .
$$

In particular, $\left(x_{n}\right)$ strongly converges towards a fixed point of $T$ provided that $\left(z_{n}\right)$ does. The latter is known to be the case e.g. for reflexive Banach spaces $X$ with uniformly Gâteaux differentiable norm provided that $T$ has a fixed point (or $C$ being bounded) and every nonempty bounded closed convex subset of $X$ has the fixed point property for nonexpansive self-mappings (see [12,13]). So, in particular, $\left(z_{n}\right)$ (and consequently $\left(x_{n}\right)$ ) strongly converges to a fixed point of $T$ if $X$ is a uniformly smooth Banach space, $T$ has a fixed point and $C$ is closed and convex (see Corollary 11.8 in [5]).

In [10], we extracted from the proof in [6] explicit and highly uniform rates of convergence for $\left\|x_{n}-T x_{n}\right\| \rightarrow 0$ (asymptotic regularity) and $\left\|x_{n}-z_{n-1}\right\| \rightarrow 0$.

Effective uniform rates on the strong convergence of $\left(z_{n}\right)$, however, in general do not exist even in the special case of Hilbert spaces. Nevertheless, one can obtain effective uniform rates $\Phi$ of so-called metastability in the sense of Tao, i.e. (here $[n ; n+g(n)]:=\{n, n+1, n+2, \ldots, n+g(n)\})$

$$
\forall \varepsilon>0 \forall g: \mathbb{N} \rightarrow \mathbb{N} \exists n \leq \Phi(\varepsilon, g) \forall i, j \in[n ; n+g(n)]\left(\left\|z_{i}-z_{j}\right\|<\varepsilon\right),
$$

which we extract for the Hilbert space case. We then combine this with our asymptotic regularity rate to obtain (again for Hilbert spaces) a rate of metastability $\Omega$ for $\left(x_{n}\right)$, in fact we get

(1) $\forall \varepsilon>0 \forall g: \mathbb{N} \rightarrow \mathbb{N} \exists n \leq \Omega(\varepsilon, g) \forall i, j \in[n ; n+g(n)] \forall l \geq n\left(\left\|x_{i}-x_{j}\right\|<\varepsilon \wedge\left\|T x_{l}-x_{l}\right\|<\varepsilon\right)$. 
Here $\Omega$ only depends (in addition to $\varepsilon, g$ ) on a Lipschitz constant $L$ for $T$, an upper bound $d \geq\left\|x_{1}-p\right\|$ for some $T$-fixed point $p$ and some moduli related to the scalars $\left(\lambda_{n}\right),\left(\theta_{n}\right)$.

(1) trivially implies the finitary (in the sense that only a finite initial segment of $\left(x_{n}\right)$ is mentioned) statement

(2) $\forall \varepsilon>0 \forall g: \mathbb{N} \rightarrow \mathbb{N} \exists n \leq \Omega(\varepsilon, g) \forall i, j \in[n ; n+g(n)]\left(\left\|x_{i}-x_{j}\right\|<\varepsilon \wedge\left\|T x_{i}-x_{i}\right\|<\varepsilon\right)$

which - in turn - trivially implies that $\left(x_{n}\right)$ strongly converges to a fixed point of $T$ as metastability ineffectively is equivalent to the usual Cauchy property. In this sense, our quantitative form also constitutes a finitary version (in the sense of Tao $[14,15]$ ) of that strong convergence theorem.

\section{Quantitative Analysis}

\subsection{Resolvent Convergence}

The following result is closely related to results of Browder [1] and Bruck [4]. It has been shown by Lan and $\mathrm{Wu}$ in [11] using techniques similar to those of Browder [2]. Although Browder's proof (for the nonexpansive case) has been analyzed by Kohlenbach in [9], it is considerably more difficult to treat than our proof below which follows the ideas of [4] (which in turn is based on [7]).

Theorem 2.1. Let $H$ be a Hilbert space, $C \subseteq H$ be a nonempty bounded closed convex subset and $T: C \rightarrow C$ be a demicontinuous pseudocontraction. Then, for each $x \in C$ and $t \in[0,1)$, there exists a unique path $\left(z_{t}\right)$ in $C$ such that $z_{t}=t T z_{t}+(1-t) x$. Moreover, the strong

$$
\lim _{t \rightarrow 1^{-}} z_{t}=z
$$

exists and is the fixed point of $T$ closest to $x$.

Proof. For each $x \in C$ and nonnegative $t<1$, the mapping $T_{t}: C \rightarrow C, z \mapsto t T z+(1-t) x$ satisfies

$$
\begin{aligned}
\left\langle T_{t} x_{1}-T_{t} x_{2}, x_{1}-x_{2}\right\rangle & =\left\langle t T x_{1}+(1-t) x-t T x_{2}-(1-t) x, x_{1}-x_{2}\right\rangle \\
& =t\left\langle T x_{1}-T x_{2}, x_{1}-x_{2}\right\rangle \\
& \leq t\left\|x_{1}-x_{2}\right\|^{2}
\end{aligned}
$$

Therefore, $T_{t}$ is pseudocontractive. It is also demicontinuous: for any sequence $\left(x_{n}\right)$ in $C$ with $x_{n} \rightarrow x$, we have

$$
\left\langle y, T_{t} x_{n}-T_{t} x\right\rangle=t\left\langle y, T x_{n}-T x\right\rangle \rightarrow 0 \text { for all } y \in H
$$

since $T$ was demicontinuous. We conclude by Corollary 4 of [4] that $T_{t}$ has a fixed point $z_{t} \in C$, i.e., a point satisfying the equation

$$
z_{t}=t T z_{t}+(1-t) x
$$

Moreover, by (1), $T_{t}$ is even strongly pseudocontractive, so $z_{t}$ is unique. To see this, suppose that $z_{t}$ and $z_{t}^{\prime}$ are two fixed points of $T_{t}$. Then, by (1),

$$
\left\|z_{t}-z_{t}^{\prime}\right\|^{2}=\left\langle z_{t}-z_{t}^{\prime}, z_{t}-z_{t}^{\prime}\right\rangle=\left\langle T_{t} z_{t}-T_{t} z_{t}^{\prime}, z_{t}-z_{t}^{\prime}\right\rangle \leq t\left\|z_{t}-z_{t}^{\prime}\right\|^{2} .
$$

Since $t<1$, this implies $z_{t}=z_{t}^{\prime}$. That $\left(z_{t}\right)$ is continuous in $t$ follows as in [12].

Strong convergence of $\left(z_{t}\right)$ will be established in the course of the proof of Theorem 2.3. That the strong limit is a fixed point of $T$ follows from (here we use that $C$ is bounded)

$$
\left|\left\langle T z_{t}-z_{t}, T z-z\right\rangle\right| \leq\left\|T z_{t}-z_{t}\right\| \cdot\|T z-z\| \stackrel{t \rightarrow 1^{-}}{\rightarrow} 0
$$


and that (using that $T$ is demicontinuous)

$$
\left\langle T z_{t}-z_{t}, T z-z\right\rangle \stackrel{t \rightarrow 1^{-}}{\rightarrow}\langle T z-z, T z-z\rangle
$$

We now proceed to show that the strong limit is the fixed point of $T$ with minimal distance from $x$. Suppose that $y$ is a fixed point of $T$. Then $y=t T y+(1-t) x$ for $t=1$. Repeating the calculations leading to (3) further below with $z_{t}=y$ and $t=1$, we obtain

$$
\|y-x\|^{2} \geq\left\|z_{s}-x\right\|^{2}+\left\|y-z_{s}\right\|^{2}, \quad \text { for all } 0<s<1 .
$$

Taking the strong limit $s \rightarrow 1$ implies

$$
\|y-x\|^{2} \geq\|z-x\|^{2}+\|y-z\|^{2}
$$

showing that $z$ is the (unique) fixed point of $T$ that is closest to $x$.

In the following we present an effective rate of metastability for the strong convergence of $\left(z_{t}\right)$. Provided that we assume the existence of $\left(z_{t}\right)$ we not even need that $T$ is demicontinuous (nor that $X$ is complete or $C$ closed).

Notation: Let $f: \mathbb{N} \rightarrow \mathbb{N}$ and $n, m \in \mathbb{N}$, then $f^{(n)}(m)$ denotes the result of $n$-times applying $f$ starting from $m$, i.e. $f^{(0)}(m):=m, f^{(n+1)}(m):=f\left(f^{(n)}(m)\right)$.

$f^{M}$ denotes the function $f^{M}(n):=\max \{f(i): i \leq n\}$.

We use the following

Lemma $2.2([8])$. Let $D \in \mathbb{R}_{+}$be a nonnegative real number and $\left(a_{n}\right)$ be a nondecreasing sequence in the interval $[0, D]$, i.e. $0 \leq a_{n} \leq a_{n+1} \leq D$. Then the following holds

$$
\forall \varepsilon>0 \forall g: \mathbb{N} \rightarrow \mathbb{N} \exists n \leq \tilde{g}^{(\lceil D / \varepsilon\rceil)}(1) \forall i, j \in[n ; n+g(n)]\left(\left|a_{i}-a_{j}\right| \leq \varepsilon\right),
$$

where $\tilde{g}(n):=n+g(n)$. Moreover, $n$ can be taken as $\tilde{g}^{(i)}(1)$ for some suitable $i \leq\lceil D / \varepsilon\rceil$.

Theorem 2.3. Let $X$ be a real inner product space and $C \subseteq X$ be a convex subset. Let $T: C \rightarrow C$ be a pseudocontraction which possesses a fixed point $v \in C$. Let $x \in C$ and assume that there exists $\left(z_{t}\right)$ for $x$ such that

$$
z_{t}=t T z_{t}+(1-t) x, \quad t \in[0,1) .
$$

Let $\left(t_{n}\right)$ be a sequence in $(0,1)$ that converges towards 1 and $h: \mathbb{N} \rightarrow \mathbb{N}$ be such that $t_{n} \leq 1-\frac{1}{h(n)+1}$ for all $n \in \mathbb{N}$. Set $z_{n}:=z_{t_{n}}$. Then, for all $\varepsilon>0$, all $g: \mathbb{N} \rightarrow \mathbb{N}$ and all $\mathbb{N} \ni d \geq\|v-x\|$

$$
\exists n \leq \Phi\left(\varepsilon, g, \chi_{g}, h, d\right) \forall i, j \in[n ; n+g(n)]\left(\left\|z_{i}-z_{j}\right\| \leq \varepsilon\right),
$$

where

$$
\Phi\left(\varepsilon, g, \chi_{g}, h, d\right):=\chi_{g}^{M}\left(g_{h, \chi_{g}}^{\left(\left\lceil 16 d^{2} / \varepsilon^{2}\right\rceil\right)}(1)\right)
$$

with

$$
g_{h, \chi_{g}}(n):=\max \left\{h(i): i \leq \chi_{g}(n)+g\left(\chi_{g}(n)\right)\right\}
$$

and $\chi_{g}: \mathbb{N} \rightarrow \mathbb{N}$ is any function satisfying

$$
\forall n \in \mathbb{N} \forall i \in\left[\chi_{g}(n) ; \tilde{g}\left(\chi_{g}(n)\right)\right]\left(\left|1-t_{i}\right| \leq \frac{1}{n+1}\right)
$$

If $\left(t_{n}\right)$ is a nondecreasing sequence in $(0,1)$ (not necessarily converging towards 1 ), then the bound can be simplified to $\Psi(\varepsilon, g, d):=\tilde{g}^{\left(\left\lceil 4 d^{2} / \varepsilon^{2}\right\rceil\right)}(1)$, where $\tilde{g}(n):=n+g(n)$. 
Proof. Assume that $z_{t} \in C$ satisfies the equation

$$
z_{t}=t T z_{t}+(1-t) x
$$

for all $t \in[0,1)$. For $1>t>s>0$, we carry out a calculation similar to [9] and [7]; Since $T z_{t}=\frac{1}{t} z_{t}-\frac{1-t}{t} x$ and $T$ is pseudocontractive,

$$
\begin{aligned}
\left\|z_{t}-z_{s}\right\|^{2} \geq\left\langle T z_{t}-T z_{s}, z_{t}-z_{s}\right\rangle & =\left\langle\frac{1}{t} z_{t}-\frac{1-t}{t} x-\frac{1}{s} z_{s}+\frac{1-s}{s} x, z_{t}-z_{s}\right\rangle \\
& =\left\langle\frac{1}{t} z_{t}-\frac{1}{t} z_{s}+\frac{1}{t} z_{s}-\frac{1}{s} z_{s}, z_{t}-z_{s}\right\rangle+\frac{t-s}{t s}\left\langle x, z_{t}-z_{s}\right\rangle \\
& =\frac{1}{t}\left\|z_{t}-z_{s}\right\|^{2}+\left\langle\frac{s-t}{s t} z_{s}, z_{t}-z_{s}\right\rangle+\frac{t-s}{t s}\left\langle x, z_{t}-z_{s}\right\rangle,
\end{aligned}
$$

and since $0<t<1$,

$$
\left\langle\frac{t-s}{s t} z_{s}, z_{t}-z_{s}\right\rangle \geq\left(\frac{1}{t}-1\right)\left\|z_{t}-z_{s}\right\|^{2}+\frac{t-s}{t s}\left\langle x, z_{t}-z_{s}\right\rangle \geq \frac{t-s}{t s}\left\langle x, z_{t}-z_{s}\right\rangle .
$$

Since $s<t$, we conclude

$$
\left\langle z_{s}-x, z_{t}-z_{s}\right\rangle \geq 0
$$

Therefore,

$$
\begin{aligned}
\left\|z_{t}-x\right\|^{2}=\left\langle z_{t}-x, z_{t}-x\right\rangle & =\left\langle z_{s}-x+\left(z_{t}-z_{s}\right), z_{s}-x+\left(z_{t}-z_{s}\right)\right\rangle \\
& =\left\langle z_{s}-x, z_{s}-x\right\rangle+\left\langle z_{t}-z_{s}, z_{t}-z_{s}\right\rangle+2\left\langle z_{s}-x, z_{t}-z_{s}\right\rangle \\
& \geq\left\|z_{s}-x\right\|^{2}+\left\|z_{t}-z_{s}\right\|^{2} .
\end{aligned}
$$

Therefore, $\left(\left\|z_{t}-x\right\|^{2}\right)_{t}$ is nondecreasing (as $\left.t \nearrow 1^{-}\right)$and

$$
\left\|z_{t}-z_{s}\right\|^{2} \leq\left|\left\|z_{s}-x\right\|^{2}-\left\|z_{t}-x\right\|^{2}\right| .
$$

$\left(z_{t}\right)$ is also bounded as follows from the existence of a fixed point $v \in C$ reasoning as in Proposition 2(iv) of [12]: If $v \in F(T)$, then

$$
\begin{aligned}
\left\|z_{t}-v\right\|^{2} & =\left\langle t T z_{t}+(1-t) x-v, z_{t}-v\right\rangle \\
& =t\left\langle T z_{t}-T v, z_{t}-v\right\rangle+(1-t)\left\langle x-v, z_{t}-v\right\rangle \\
& \leq t\left\|z_{t}-v\right\|^{2}+(1-t)\left\langle x-v, z_{t}-v\right\rangle
\end{aligned}
$$

which implies

$$
(1-t)\left\|z_{t}-v\right\|^{2} \leq(1-t)\|x-v\| \cdot\left\|z_{t}-v\right\| .
$$

Since $t<1$, this implies that $\left\|z_{t}-v\right\| \leq\|x-v\|$. Hence

$$
\left\|z_{t}-x\right\| \leq\left\|z_{t}-v\right\|+\|v-x\| \leq 2\|v-x\| \leq 2 d, \text { i.e. }
$$

$\left(\left\|z_{t}-x\right\|^{2}\right)_{t}$ is bounded by $4 d^{2}$.

Together with Lemma 2.2 applied to $\left(\left\|z_{t_{n}}-x\right\|^{2}\right)_{n}, 4 d^{2}$ and $\varepsilon^{2}$ and (4) above the theorem now follows in the case where $1>t_{n+1} \geq t_{n}>0$ for all $n \in \mathbb{N}$. For the case of a general sequence $\left(t_{n}\right)$ which is assumed to converge to 1 one reasons literally as in the proof of Theorem 4.2 in [9].

Remark 2.4. Theorem 4.2 of [9] establishes the same result for nonexpansive mappings.

Remark 2.5. It is not hard to show that Theorem 2.3 also holds with the assumption $F(T) \neq \emptyset$ being replaced by $\forall \varepsilon>0 \exists v_{\varepsilon} \in C\left(\left\|x-v_{\varepsilon}\right\| \leq d \wedge\left\|T v_{\varepsilon}-v_{\varepsilon}\right\| \leq \varepsilon\right)$. 


\subsection{Asymptotic Regularity of the Bruck Iteration}

Theorem 2.6 ([10]). Let $C$ be a nonempty, closed and convex subset of a real Banach space $X$ and $x \in C$. Let $T: C \rightarrow C$ be a Lipschitzian pseudocontractive mapping with Lipschitz constant $L$ and for some $d>0$ assume that $T$ possesses arbitrarily good $\varepsilon$-fixed points $p_{\varepsilon} \in C$ with $\left\|x-p_{\varepsilon}\right\|<$ d. Let $\left(x_{n}\right)$ be the Bruck iteration (Definition 1.1) with starting point $x_{1}:=x$. Let $z_{n}$ be the unique element in $C$ satisfying $z_{n}=t_{n} T\left(z_{n}\right)+\left(1-t_{n}\right) x_{1}$ with $t_{n}:=1 /\left(1+\theta_{n}\right)$. Given rates of convergence/divergence $R_{i}:(0, \infty) \rightarrow \mathbb{N}$ for the Chidume-Zegeye conditions 1.2, we get

$$
\forall \varepsilon>0 \forall n \geq \Psi\left(d, L, R_{1}, R_{2}, R_{3}, R_{4}, \varepsilon\right)\left(\left\|x_{n}-T x_{n}\right\|<\varepsilon\right)
$$

and

$$
\forall \varepsilon>0 \forall n \geq \chi\left(d, L, R_{1}, R_{2}, R_{3}, R_{4}, \varepsilon\right)\left(\left\|x_{n}-z_{n-1}\right\|<\varepsilon\right)
$$

where

$$
\Psi\left(d, L, R_{1}, R_{2}, R_{3}, R_{4}, \varepsilon\right)=\max \left\{N_{2}(C)+1, R_{1}\left(\frac{\varepsilon}{4 r}\right)+1\right\}
$$

and

$$
\chi\left(d, L, R_{1}, R_{2}, R_{3}, R_{4}, \varepsilon\right)=N_{2}(C)+1
$$

with

$$
\begin{aligned}
& N_{1}(\varepsilon):=\max \left\{R_{3}\left(\frac{2 \varepsilon s}{3 r^{2}}\right), R_{4}\left(\sqrt{\frac{\varepsilon}{r^{2}}+\frac{9}{4}}-\frac{3}{2}\right)\right\}, \\
& N_{2}(x):=R_{2}\left(\frac{x}{2}\right)+1 \\
& C:=\frac{8(1+L)^{2} r^{2}}{\varepsilon^{2}}+2\left(N_{1}\left(\frac{\varepsilon^{2}}{8(1+L)^{2}}\right)-1\right), \\
& r:=\max \left\{\frac{(2+L)^{R_{3}(d)}-1}{1+L} d, 2 d\right\} \\
& s:=\frac{1}{2\left(\frac{5}{2}+L\right)(2+L)} .
\end{aligned}
$$

Proof. The first claim is Theorem 1 in [10] and the second claim follows from formula (24) in the proof of that theorem (even with $\varepsilon$ being replaced by $\varepsilon /(2(1+L))$ in the definition of $\chi$ ).

Corollary 2.7 ( [10]). In the situation of Theorem 2.6, one may drop the condition that $T$ has arbitrarily good approximate fixed points and instead require $\operatorname{diam}(C) \leq d$. In this case,

$\chi\left(d, L, R_{1}, R_{2}, R_{3}, R_{4}, \varepsilon\right):=N_{2}(C)+1$ and $\Psi\left(d, L, R_{1}, R_{2}, R_{3}, R_{4}, \varepsilon\right)=\max \left\{\chi(\varepsilon), R_{1}\left(\frac{\varepsilon}{2 d}\right)+1\right\}$

and

$$
\begin{aligned}
& N_{1}(\varepsilon):=\max \left\{R_{3}\left(\frac{\varepsilon}{4 d^{2}(2+L)}\right), R_{4}\left(\sqrt{\frac{\varepsilon}{d^{2}}+1}-1\right)\right\} \\
& N_{2}(x):=R_{2}\left(\frac{x}{2}\right)+1 \\
& C:=\frac{8(1+L)^{2} d^{2}}{\varepsilon^{2}}+2\left(N_{1}\left(\frac{\varepsilon^{2}}{8(1+L)^{2}}\right)-1\right) .
\end{aligned}
$$




\subsection{Strong Convergence of the Bruck Iteration}

Theorem 2.8. If, in the situation of Theorem 2.6, $X$ is a Hilbert space, then (assuming w.l.o.g. $L \geq 1$ ) for all $\varepsilon>0$ and all $g: \mathbb{N} \rightarrow \mathbb{N}$

$\exists n \leq \chi^{M}\left(g_{h, \chi}^{\left(\left\lceil 64 d^{2} / \varepsilon^{2}\right\rceil\right)}(1)\right)+\Psi(\varepsilon)+1 \forall i, j \in[n ; n+g(n)] \forall l \geq n\left(\left\|x_{i}-x_{j}\right\| \leq \varepsilon \wedge\left\|T x_{l}-x_{l}\right\| \leq \varepsilon\right)$ where $h: \mathbb{N} \rightarrow \mathbb{N}$ is a function such that $h(n) \geq 1 / \theta_{n}$ for all $n \in \mathbb{N}$ and $\chi(n):=R_{1}(1 / n)$,

$$
g^{\prime}(n):=g(n+1+\Psi(\varepsilon))+\Psi(\varepsilon)+1, \quad g_{h, \chi}(n):=\max \left\{h(i): i \leq \chi(n)+g^{\prime}(\chi(n))\right\},
$$

and $R_{1}$ and $\Psi$ as in Corollary 2.7 .

Proof. In Theorem 2.6, the resolvent $z_{t}$ is instantiated with the sequence $t=t_{n}=\frac{1}{1+\theta_{n}}$ and the starting point $x_{1}$. We now show how to apply Theorem 2.3 to this instantiation; if we set $\chi(n):=R_{1}(1 / n)$, then $\theta_{i} \leq 1 / n$ for all $i \geq \chi(n)$. Since $\theta_{n} \in(0,1]$, this implies

$$
\left|1-t_{i}\right|=1-\frac{1}{1+\theta_{i}} \leq 1-\frac{1}{1+\frac{1}{n}}=\frac{1}{n+1}, \quad \text { for all } i \geq \chi(n) .
$$

Since this holds for all $i \geq \chi(n)$, the function $\chi$ satisfies (2) independently of the counter-function $g$ and we may set $\chi_{g}:=\chi$ in Theorem 2.3 .

Moreover, for all $n \in \mathbb{N}, h(n) \geq 1 / \theta_{n}$ implies $1+h(n) \geq \frac{1+\theta_{n}}{\theta_{n}}$, whence

$$
\frac{1}{h(n)+1} \leq \frac{\theta_{n}}{1+\theta_{n}}=1-\frac{1}{1+\theta_{n}}
$$

Therefore,

$$
t_{n}=\frac{1}{1+\theta_{n}} \leq 1-\frac{1}{h(n)+1}, \quad \text { for all } n \in \mathbb{N} .
$$

Now observe that, by Theorem 2.3 and Remark 2.5 applied to the counter-function $g^{\prime}$ and error $\varepsilon / 2$, there exists an $n \leq \chi^{M}\left(g_{h, \chi}^{\left(\left\lceil 64 d^{2} / \varepsilon^{2}\right\rceil\right)}(1)\right)$ such that

$$
\left\|z_{i}-z_{j}\right\| \leq \frac{\varepsilon}{2}, \quad \text { for all } i, j \in\left[n ; n+g^{\prime}(n)\right] .
$$

Since $\left[n ; n+g^{\prime}(n)\right]=[n ; n+1+\Psi(\varepsilon)+g(n+1+\Psi(\varepsilon))] \supseteq[n+\Psi(\varepsilon) ; n+1+\Psi(\varepsilon)+g(n+1+\Psi(\varepsilon))]$, we conclude that if we set $n_{0}:=n+1+\Psi(\varepsilon)$, then

$$
\left\|z_{i-1}-z_{j-1}\right\| \leq \frac{\varepsilon}{2}, \quad \text { for all } i, j \in\left[n_{0} ; n_{0}+g\left(n_{0}\right)\right]
$$

Since $n_{0} \geq \Psi(\varepsilon)$, we conclude from (24) of [10] for all $n \geq n_{0},\left\|x_{n}-z_{n-1}\right\| \leq \frac{\varepsilon}{2(1+L)} \leq \varepsilon / 4$, since we may w.l.o.g. assume $L \geq 1$. Thus,

$$
\left\|x_{i}-x_{j}\right\| \leq\left\|x_{i}-z_{i-1}\right\|+\left\|z_{i-1}-z_{j-1}\right\|+\left\|z_{j-1}-x_{j}\right\| \leq \varepsilon, \quad \text { for all } i, j \in\left[n_{0} ; n_{0}+g\left(n_{0}\right)\right] .
$$

Moreover, we get from Theorem 2.6

$$
\left\|x_{n}-T x_{n}\right\| \leq \varepsilon, \quad \text { for all } n \geq \Psi(\varepsilon) .
$$

This completes the proof.

Corollary 2.9. If $\left(\theta_{n}\right)$ is nondecreasing, then for all $\varepsilon>0$ and $g: \mathbb{N} \rightarrow \mathbb{N}$

$$
\exists n \leq \tilde{g}^{\prime\left(\left\lceil 16 d^{2} / \varepsilon^{2}\right\rceil\right)}(1)+\Psi(\varepsilon)+1 \forall i, j \in[n ; n+g(n)] \forall l \geq n\left(\left\|x_{i}-x_{j}\right\| \leq \varepsilon \wedge\left\|T x_{l}-x_{l}\right\| \leq \varepsilon\right)
$$

where $\tilde{g}^{\prime}(n)=g^{\prime}(n)+n$ and $g^{\prime}(n)=g(n+1+\Psi(\varepsilon))+\Psi(\varepsilon)+1$. 
Proof. Since $\left(\theta_{n}\right)$ is nondecreasing, the second part of Theorem 2.3 implies that there exists an $n \leq \tilde{g}^{\prime\left(\left\lceil 16 d^{2} / \varepsilon^{2}\right\rceil\right)}(1)$ such that

$$
\left\|z_{i}-z_{j}\right\| \leq \frac{\varepsilon}{2}, \quad \text { for all } i, j \in\left[n ; n+g^{\prime}(n)\right],
$$

which is the analog to equation (5). The remainder of the proof is then the same.

As a corollary to the proof of Theorem 2.8 we get the following transformation of an assumed rate of metastability for $\left(z_{n}\right)$ into one for $\left(x_{n}\right)$ in general Banach spaces:

Corollary 2.10. In the situation of Theorem 2.6 (so $X$ is not necessarily a Hilbert space), suppose that for all $g: \mathbb{N} \rightarrow \mathbb{N}$ and $\varepsilon>0$,

$$
\exists n \leq \Omega(d, g, \varepsilon) \forall i, j \in[n ; n+g(n)]\left(\left\|z_{i}-z_{j}\right\| \leq \varepsilon\right),
$$

and let $\chi^{M}(n):=R_{1}(1 / n)$. Then, for all $\varepsilon>0$ and $g: \mathbb{N} \rightarrow \mathbb{N}$,

$\exists n \leq \chi^{M}(\Omega(d, g, \varepsilon / 2))+\Psi(\varepsilon)+1 \forall i, j \in[n ; n+g(n)] \forall l \geq n\left(\left\|x_{i}-x_{j}\right\| \leq \varepsilon \wedge\left\|T x_{l}-x_{l}\right\| \leq \varepsilon\right)$.

Remark 2.11. For the canonical choice $\lambda_{n}=\frac{1}{(n+1)^{a}}$ and $\theta_{n}=\frac{1}{(n+1)^{b}}$, where $0<b<a$ and $a+b<1$, the bound is as stated in Corollary 2.9 .

\section{References}

[1] F. E. Browder. Existence of Periodic Solutions for Nonlinear Equations of Evolution. Proceedings of the National Academy of Sciences, 53:1100-1103, 1965.

[2] F. E. Browder. Convergence of approximants to fixed points of nonexpansive nonlinear mappings in Banach spaces. Archive for Rational Mechanics and Analysis, 240:82-90, 1967.

[3] F. E. Browder and W. V. Petryshyn. Construction of fixed points of nonlinear mappings in Hilbert spaces. Journal of Mathematical Analysis and Applications, 20:197-228, 1967.

[4] R. E. Bruck. A strongly convergent iterative solution of $0 \in U(x)$ for a maximal monotone operator $U$ in Hilbert space. Journal of Mathematical Analysis and Applications, 48:114-126, 1974.

[5] C. Chidume. Geometric Properties of Banach Spaces and Nonlinear Iterations. Springer Lecture Notes in Mathematics, 2009.

[6] C. E. Chidume and H. Zegeye. Approximate fixed point sequences and convergence theorems for Lipschitz pseudocontractive maps. Proceedings of the American Mathematical Society, 132:831-840, 2004.

[7] B. Halpern. Fixed points of nonexpanding maps. Bulletin of the American Mathematical Society, 73:957-961, 1967.

[8] U. Kohlenbach. Arithmetizing proofs in analysis. In: Larrazabal, J.M., Lascar, D., Mints, G. (eds.), Logic Colloquium '96, Springer Lecture Notes in Logic, 12:115-158, 1998.

[9] U. Kohlenbach. On quantitative versions of theorems due to F.E. Browder and R. Wittmann. Advances in Mathematics, 226:2764-2795, 2011.

[10] D. Körnlein and U. Kohlenbach. Effective rates of convergence for Lipschitzian pseudocontractive mappings in general Banach spaces. Nonlinear Analysis, 74:5253-5267, 2011.

[11] K. Q. Lan and J. H. Wu. Convergence of approximants for demicontinuous pseudo-contractive maps in Hilbert spaces. Nonlinear Analysis, 49:737-746, 2000. 
[12] C. H. Morales and J. S. Jung. Convergence of paths for pseudocontractractive mappings in Banach spaces. Proceedings of the American Mathematical Society, 128:3411-3419, 2000.

[13] S. Reich. Strong convergence theorems for resolvents of accretive operators in Banach spaces. Journal of Mathematical Analysis and Applications, 75:287-292, 1980.

[14] T. Tao. Soft analysis, hard analysis, and the finite convergence principle. Essay posted May 23, 2007. Appeared in: T. Tao, Structure and Randomness: Pages from Year One of a Mathematical Blog. AMS, 298pp., 2008'.

[15] T. Tao. Norm convergence of multiple ergodic averages for commuting transformations. Ergodic Theory and Dynamical Systems, 28:657-688, 2008. 